\title{
Polio Cost Effectiveness in Nigeria; A Lesson to Be Learnt
}

\author{
S. M. ZEESHAN QADAR* \\ Bachelor of Pharmacy University of Karachi, Master of Philosophy in Pharmacognosy (course work completed), Master of Science \\ International Health Policy and Management Brandeis University, Doctoral Student (Public Health Education) Southern Illinois \\ University Carbondale \\ *Corresponding author: zeeshanqadarpk@gmail.com
}

Received October 15, 2014; Revised November 20, 2014; Accepted December 04, 2014

\begin{abstract}
This review paper highlights the importance of a cost effective intervention in global polio eradication fight for developing nations. The main aim of this paper is to propose an intervention which is highly cost effective for Nigeria where polio is still prevalent. This paper examines cost effectiveness analysis of a feign one-year vaccination intervention using the oral polio vaccine (OPV) in Kano State, Nigeria. To establish cost-effectiveness healthy days saved by this vaccination intervention were used instead of Disability Adjusted Life Years (DALYS). In this case cost effectiveness was established by dividing intervention cost (\$1.7 million) to the number of healthy days saved (\$2485) to get cost per day of healthy life (\$472). This cost effective intervention gives about \$472 cost per day of healthy life which was then compared with the GDP per income of Kano (\$1288). It was evident from the results of the study that as the cost of the intervention is below the GDP per capita of Kano, Nigeria which means this method is highly cost efficient. The cost effectiveness of this intervention implies that the program of vaccination is economically viable and should therefore be encouraged, than just concentrating on treating polio. With the economic crunch times in the world, there is a strong urge for cost effective programs to address for deadly diseases and the evaluated intervention would be one of them.
\end{abstract}

Keywords: developing nations, polio, Kano, Nigeria, OPV (Oral Polio Vaccine), small pox

Cite This Article: S. M. ZEESHAN QADAR, "Polio Cost Effectiveness in Nigeria; A Lesson to Be Learnt." American Journal of Pharmacological Sciences, vol. 2, no. 5B (2014): 4-7. doi: 10.12691/ajps-2-5B-2.

\section{Introduction}

Poliomyelitis is a disease responsible for significant number of mortalities and morbidities mostly in children under 5 years of age. The residual effects of polio are seen long after the acute phase of the disease has ended, with paralysis lasting for the lifetime of the affected individual. It is a contagious, historically devastating infectious disease caused by a virus that lives in the throat and intestinal tract and most often spread through person-toperson contact with the fecal matter of an infected person and may also spread through oral or nasal secretions. It results in disability and death for those who develop symptoms of the disease, and it is the common cause of disability among children under 15 years (WHO, 1994).

Although virtually eliminated from the Western hemisphere in the second half of the 20th century through the use of routine immunization, Nigeria, along with Afghanistan, India, and Pakistan are the four countries that have never interrupted polio virus transmission and therefore remains a significant public health problem (Global Polio Eradication Initiative Monthly Situation Report, December 2007). In Nigeria, several attempts at eradicating polio have been attempted, with the major problem being compliance with immunization programs in the northern parts of the country. In 2008, about 806 new cases of polio were diagnosed (WHO Polio case count, 2009). Globally 650 polio cases in 2011 were reported and WHO has been focused on global polio eradication by 2015 (polio eradication, 2012)

It is therefore imperative to eliminate this crippling and potentially fatal virus and make poliomyelitis only the second disease in the history of humankind to be eradicated (smallpox being the only one so far). To keep this study real time some of the background data was based on the paper by Kahn and Ehrett (2003) and Kahn (2010) which had focused on cost and effectiveness of polio immunization and eradication activities from long-term global perspective. While these articles used Disability Adjusted Life Years (DALYs) as the measure of effectiveness, this paper used the number of healthy days saved by is vaccination intervention as the measure of effectiveness instead. The cost effectiveness for a polio eradication program in Kano State, Nigeria, was analyzed using the Kahn article as the background. The costeffectiveness of an OPV (oral polio vaccine) vaccination intervention in Kano, where polio still has a significant effect was focused. OPV is the vaccine of choice in countries where polio is still uninterrupted. The cost of this one-year intervention is compared with the healthy days saved by this intervention. The cost of one year intervention to the Gross Domestic Product (GDP) of 
Kano to determine if it is cost effective or not was compared. From 2008 estimates, GDP per capita of Kano is $\$ 1288$ (Can Bank Global income Distribution Database, 2008). Nigeria is one of the countries in which polio is endemic, about $30 \%$ of the total number of cases around the world were from Nigeria. According to 2008 data, 242 cases were reported only from Nigeria (WHO, 2008). The data is clearly indicative that polio is still a threat to global citizens and especially in Nigeria. With the prevalence of this deadly disease in Nigeria and other developing nations, cost of the programs to eradicate polio is always a daunting subject when designing an intervention.

\section{Methodology}

Children less than 5 years of age in Kano state is the focus population of this paper. These are the ages most susceptible to the disease. Most recent information on the disease is used and where information was unavailable, some assumptions were made in the article. This analysis assumed that the coverage by polio vaccination is $100 \%$ and all the conditions are favorable for this intervention. The total population of children under the age of five is about 1.45 million children. This was calculated using a comparable immunization program in Nigeria and scaling down the cost to the population of Kano (9.4 million people). The cost effectiveness perspective of polio eradication program had been previously documented in several studies. The total cost presumed for this study is to be $\$ 1.7$ million, which includes the cost of the OPV, which is about 8 cents for a single dose (Global Polio eradication Initiative website, 2009), transportation, delivery costs, storage, maintenance, training vaccinators, mobilization, and also monitoring and surveillance.

The effectiveness data was calculated using the number of healthy days of life saved by this vaccination intervention. The difference between this figure and the healthy days lost without the intervention to get the number of healthy days saved by this intervention were calculated. This is very important because with healthy lives saved, the quality of life improves significantly; children would lead healthy lives and therefore be more productive in society in the future. After this, the cost per year of life saved is calculated and compared to the Gross Domestic Product (GDP) of Kano to determine if the intervention is cost effective. From the past several years developing world has been looking to design an efficient intervention which is beneficial for health and is cost effective. Hence, this paper is designed to bring a viable low cost option to International community for polio eradication. Finally, a sensitivity analysis was done to depict how a variation in some of the costs affects the effectiveness of the intervention on the total cost and the variation in total cost. A change in the unit cost of vaccination was assumed and analyzed how this affects viability of the intervention.

\subsection{Simplifying Assumptions}

Several assumptions were made to make the calculations more feasible. These include the following,

i. The vaccination intervention will be for a period of one-year. ii. The vaccination would be effective for the lifetime of the patient.

iii. The average morbidity from polio is the entire lifetime (since the disability that arises is irreversible).

iv. Immunization of all children throughout the state would be feasible, without geographical access problems and would have $100 \%$ coverage.

v. The target population for this intervention would be compliant with the vaccination and will not resist.

vi. That of the 806 cases of polio recorded in Nigeria in 2001, Kano state had about 240 cases.

vii. That Kano has the same proportion of children less than 5 years as Nigeria (i.e. $17.5 \%$ of the population).

viii. Most children would contract the disease at around 4 years old. Therefore subtracting this from the life expectancy at that age (52.6 years), gives 48.6 years of disability.

ix. OPV vaccine is $100 \%$ effective, therefore after our vaccination, there will be no more cases of polio.

\subsection{Cost Analysis}

In calculating the total cost of the intervention for the entire Nigerian population under 5 years, several factors had to be taken into account. The first consideration was the total costs associated for providing the polio vaccine to the current vaccination program in the Kano, Nigeria. Instead of creating an independent network to distribute the vaccine, it seems more efficient to include Polio in the existing vaccination program and adjust the program accordingly.

The elements of the intervention considered in the cost are distribution, delivery, storage, training and social mobilization. The costs of these elements are presented on per vaccination basis. Total costs are then extrapolated to the under five population of Kano. An assumption was made that a vaccination study in Tanzania, with a GDP of $\$ 1400$ (CIA Fact book), would have similar costs to Kano. Therefore, the cost for the intervention was calculated to the population of Kano.

\subsection{Distribution}

There is a vaccination station in Kano State and was assumed as intervention site. The costs associated with distribution include freight charges, which include costs for vehicles, fuel, personnel to drive trucks, purchasing cold boxes for effective transportation of vaccines (cold chain), and also for air-fare fees for areas inaccessible by land. Transportation, delivery and distribution costs were therefore about U.S. \$0.39 per vial.

\subsection{Storage}

The vaccines need to be stored properly at the appropriate temperatures to prevent damage. The storage costs include cost of storage in cold bags, and the amount of time the vaccines will remain in storage. The cost of storage was estimated to be U.S. \$0.103 per vial.

\subsection{Training}

For the vaccine to be administered appropriately, health workers need training. This training was assumed for once. 
Training cost included payment of trainers, cost feeding etc. Cost of training supplies and stationery were also included. The total costs for training was estimated to be U.S. \$0.03 per vial.

\subsection{Social Mobilization}

Social mobilization is very important part to initiate the introduction of polio vaccine. Publicity and marketing is also essential. People need to be educated about the benefits of the intervention, and old preconception that occurred in the state about the vaccine causes sterility needs to be corrected. This will help to achieve the target $100 \%$ coverage. In a similar vaccination exercise carried out in Tanzania, the cost was about $\$ 450,000$ for mobilization, marketing and promotion. Therefore the data to fit the population of Nigeria was extrapolated and then scaled down to the population of Kano. About U.S. \$0.11 per vaccination in Kano was calculated (Table 1).

Table 1. Cost of Vaccination

\begin{tabular}{ccc}
\hline Parameters & $\begin{array}{c}\text { Cost per } \\
\text { Vaccination (\$) }\end{array}$ & $\begin{array}{c}\text { Cost per population } \\
\text { (millions \$) }\end{array}$ \\
\hline Distribution and & 0.39 & 0.64 \\
Delivery & 0.10 & 0.17 \\
Storage & 0.03 & 0.05 \\
Training & 0.11 & 0.18 \\
Social Mobilization & 0.08 & 0.13 \\
Cost of OPV & 0.71 & 1.17 \\
\hline Total & &
\end{tabular}

\subsection{Cost per Vaccination}

This paper assumed 100\% effectiveness of this Polio intervention. Therefore, the total cost of the vaccination program was the total cost of vaccination. The population less than 5 years in Kano was about 1.64 million and the unit cost for one vial of polio vaccine was $\$ 0.08$, giving the total cost of polio vaccine in Kano to be $\$ 13,1371$. The unit cost of storage was $\$ 0.10$, which gave us $\$ 169,887$ the total amount for storage costs. The unit cost for transportation and delivery of Polio vaccine was \$0.39 which when multiplied by the total number of population under 5 in Kano gives us \$640,436. The unit cost for training is $\$ 0.03$ which when multiplied by the 1.64 million gave the total cost of $\$ 49,264$. The social mobilization unit cost was $\$ 0.11$ which when multiplied with the total population gaves a total of \$180,635.

\subsection{Effective Analysis}

The article by Kahn and Ehrett, 2003 analyzed the effectiveness of a polio eradication program in the USA and Italy using Disability Adjusted Life Years (DALYs) as the measure of effectiveness. However, in this paper, the number of days saved by this polio intervention program was used to assess its cost effectiveness in Kano State. This was calculated by calculating the potential healthy days saved by calculation and from this calculating the cost per healthy day saved. A theoretical vaccination program was assumed for this paper. Polio mainly affects children less than five years, therefore population under five years was the focus of this intervention. In order to analyze the effectiveness of the intervention, several parameters were required for computation. They include the case fatality of polio, the years of life lost due to premature death by polio, and the incidence rate of the disease in Kano state. Also needed was the population of children under five years in Kano state. This number was difficult to obtain, therefore we made an extrapolation from the Nigerian population, where the population less than 5 years old is $17.5 \%$ (WHOSIS, 2006). We therefore assumed that Kano state would have the same proportion and applied it to the Kano population, which is 9.38 million people (Can Bank Global Income Distribution database, 2008). The author arrived at 1.64 million children (Table 2).

Table 2. Population Parameters

\begin{tabular}{cc}
\hline Parameters & Parameter Values \\
\hline Population <5 yrs in Kano (in millions) & 1.64 \\
Case fatality (\%) & 1.00 \\
Case Survival (\%) & 99.00 \\
Number of cases surviving (\%) & 99.00 \\
Incidence rate per 1000 without vaccination & 0.15 \\
Incidence rate per 1000 with vaccination & 0.00 \\
Average duration of disability (years) & 48.60 \\
Coverage of Intervention (\%) & 100.00 \\
Efficacy of OPV (\%) & 100.00 \\
Number of Polio cases in Kano & 242.00 \\
\hline
\end{tabular}

The oral polio vaccine, which is the vaccination of choice in this paper, is assumed to have $100 \%$ efficacy. The PDLL due to death before the intervention was calculated by multiplying the case fatality (1\%), the incidence of polio per thousand (0.15), the life expectancy in days at ages less than 5 years (48.6 years) and the number of days in a year (365.25 days). Subsequently, the PDLL due to disability per a thousand population before the vaccination intervention was calculated by multiplying the incidence per thousand (0.15), proportion of people surviving (99\%), the average duration of disease (assumed to be for the lifetime of the individual i.e. 48.6 years) and the number of days in a year (365.25 days). The values were 26 and 2589 for the days lost due to death and the days lost due to disability respectively. Adding them gave 2615 days. Therefore, 2615 healthy days of life per thousand populations is lost each year due to polio. The same calculation for the number of days lost after the polio intervention was calculated about 131.3 days lost due to polio per thousand populations.

Therefore it was conclude that by subtracting the days lost after the polio intervention from the days lost in the absence of any intervention, the number of days saved by intervention in Kano state. Therefore 2485 healthy days per thousand populations was saved by this vaccination intervention in Kano state (Table 3).

Table 3. PDLL

\begin{tabular}{ccc}
\hline & Without Vaccination & With vaccination \\
\hline PDLL due to mortality & 26.00 & 1.30 \\
PDLL due to morbidity & 2589.00 & 130.00 \\
Total & 2615.00 & 131.30 \\
\hline Net PDLL & $\mathbf{2 4 8 5 . 0 0}$ & \\
\hline
\end{tabular}

\section{Results}

\subsection{Cost Effectiveness Analysis}

To analyze the cost effectiveness of this vaccination intervention, the cost and the effectiveness of obtain a cost per day of healthy life was combined. The cost of this intervention ( $\$ 1.7$ million) was divided by the number of healthy days saved (\$2485). This gave about $\$ 472$ per healthy day saved. This value was then compared with the 
GDP per income of Kano (\$1288) implies that the program of vaccination was more economically viable than just concentrating on treating polio. The vaccine is also a $100 \%$ effective and individuals are vaccinated for life (Table 4).

Table 4. Cost effectiveness

\begin{tabular}{cc}
\hline Cost of intervention (\$ millions) & 1.72 \\
Net days of life saved & 2485 \\
Cost Effectiveness (\$ per days of life saved) & 472 \\
\hline
\end{tabular}

\subsection{Sensitivity Analysis}

The sensitivity analysis for this intervention was carried out to determine at which cost of vaccination that doubts about the economic viability of the intervention would arise. Different costs per vial of OPV were used until cost that was greater than the GDP per capita of Kano was achieved. From sensitivity analysis, the cost of OPV at which the intervention was not viable was \$1.5. At this point, the intervention was not economically viable and therefore should not be undertaken. In addition, the borderline cost of a vial of OPV was (\$1.42), where the cost per days of life saved by the intervention was almost equal to the GDP of Kano. At this point, the people in authority would have to decide whether to go ahead with the intervention or not. In addition, to confirm the economic viability of this intervention sensitivity analysis was conducted to determine the cost of Oral Polio Vaccine (OPV) at different dollars. At \$1.5 per vial Oral Polio Vaccine (OPV) was not cost effective and not viable to be adopted hence with sensitivity analysis the borderline cost of a vial of OPV was (\$1.42), whereas the cost per days of life saved by the intervention was almost equal to the GDP of Kano (Table 5).

Table 5. Sensitivity Analysis

\begin{tabular}{cc} 
& Table 5. Sensitivity Analysis \\
\hline Cost per vial (\$) & Cost Effectiveness (\$ per days of life saved) \\
\hline 0.10 & 460 \\
0.50 & 712 \\
1.00 & 1025 \\
1.30 & 1213 \\
1.40 & 1276 \\
1.42 & 1289 \\
1.50 & 1339 \\
\hline
\end{tabular}

\section{Conclusion and Policy Implication}

Finally, calculating the effectiveness of this program proved somewhat difficult as several assumptions had to be made, either due to lack of data or to simplify calculations. However, the cost effectiveness of this intervention was economically viable and should therefore be encouraged, despite the low incidence of polio in the state. Although this was a hypothetical case, the threat of polio spreading worldwide is real and firm steps need to be taken to prevent this, especially in countries where compliance is the major problem. Eliminating this disease has enormous benefits to the life of not only the inhabitants of a country, but also worldwide benefits. It would also substantially decrease the number of permanently disabled people around the world. It should therefore be taken up as a challenge by health organizations around the world to rid the world of this crippling disease.

With the scarce budget of health care in developing nations especially in preventive health care, polio ranks extremely low and a negligible amount of dollars are allocated. Most of the polio initiative funding is from International donor agencies and developed nations with an aim to strengthen structural changes through which polio can be eradicated. This cost effective program could be a good investment for the developed nations. With the cost savings it brings to the table it provides an opportunity for policy designers in both developing and developed countries to consider this viable option to overcome the cost of polio interventions.

\section{Acknowledgement}

This research paper would not have been possible by the sincere and dedicated efforts from one of my good friend Dr. Ola-Dipo Fadayoumi for collecting the data from Kanu Nigeria by himself. He is a Resident Medical Officer in US and has worked in both clinical and public health in Nigeria. All hands for his contribution

\section{References}

[1] C-GIDD (CanBank Global Income Distribution database), 2008

[2] CIA: The World Factbook, 2008. Retrieved on 03/24/09 at https://www.cia.gov/library/publications/the-worldfactbook/rankorder/2004rank.html

[3] Global Polio Eradication Initiative Website, 2012. Retrieved on 03/11/12 from http://www.polioeradication.org/Dataandmonitoring/Poliothiswee k.aspx

[4] Global Polio Eradication Initiative Website, 2009. Retrieved on 03/25/09 from http://www.polioeradication.org/vaccines.asp

[5] Kahn, M, \& Ehreth, J. 2003. Costs and benefits of polio eradication: a long run global perspective. Vaccine 21: 702-705.

[6] Kahn, M, 2010. Economic Costs and Disability-Adjusted Life Years in Polio Eradication: A Long-Run Global Perspective. Springer Science Business Media LLC, 78: 1354-1371.

[7] Singh, J et al, 1992. Epidemiological evaluation of Oral polio vaccine efficacy in Delhi, India. Journal of Pediatrics 59: 321-323.

[8] Tanzania Malaria Operational Plan, 2008. Retrieved from http://www.fightingmalaria.gov/countries/mops/tanzania_mopfy08.pdf

[9] WHO Polio case count, 2009. Retrieved on 03/24/09 at http://www.polioeradication.org/content/general/casecount.pdf.

[10] WHO press release WHO/83, Nov 24, 1994.

[11] WHO Statistical Information System (WHOSIS), 2008.

[12] WHO, UNICEF, CDC, December 2007. Global Polio Eradication Initiative Monthly Situation Report. 